\title{
Spontaneous Internal Carotid Dissection in a 38 Year Old Female: A Case Report
}

\section{Kareem Abed ${ }^{*}$, Amit Misra and Viren Vankawala}

Kareem Abed, Atlanticare Regional Medical Center, USA

\begin{abstract}
This case describes a patient with amaurosis fugax as a result of non-traumatic internal carotid dissection. Typical symptoms of monocular blindness, especially in the setting of headache or neck pain should prompt evaluation for carotid dissection. Initial presentation with transient vision loss, lasting thirty minutes with a normal ophthalmic exam should raise suspicion for transient ischemic attack as well as carotid dissection. Symptoms may progress with resultant cerebral infarction as the dissection progresses. Carotid ultrasound with notable decreased internal carotid artery velocity prompts CT angiography of the neck confirming the carotid dissection. Anti-platelet therapy is the first line treatment, with anticoagulation reserved for cases of recurrent, progressive symptomatic episodes.
\end{abstract}

Keywords: Carotid dissection, Amaurosis fugax

\section{Introduction}

Carotid dissection may be traumatic or spontaneous, resulting in hemorrhage within the arterial wall. Atraumatic causes are spontaneous, resulting in the absence of an external force such as with exertion of the neck (rapid or prolonged head movement). Atraumatic dissections have a different appearance than those of trauma, as they may have involvement of the common carotid, resulting in complete occlusion and being less likely to spontaneously resolve. Systemic hypertension and arteriopathy are common causes of spontaneous carotid dissection. Arteriopathy includes fibromuscular dysplasia, Marfan's syndrome, type 4 Ehlers-Danlos syndrome, cystic medial necrosis, atheroma, and pheochromocytoma [1].

In the majority of patients with carotid dissection, symptoms include headache or neck pain. In carotid dissections involving the internal carotid artery, retro orbital headaches, with presentation similar to that of Horner's syndrome, as described in this case. Internal carotid artery dissections have also been located more frequently near the carotid bifurcation, more commonly at the supraclinoid site and cervical segments of C1-C2 [1]. In cases where primary carotid dissections are noted, careful examination for other regions is imperative as twenty percent of cases will have multiple dissections. As previously noted, hemorrhage within the carotid wall may occur, more commonly as a result of intimal injury. It is also possible this to occur due to hemorrhage from the vasa vasorum into the media. Formations of a hematoma may further lead to extensive dissection through the media resulting in narrowing of the lumen or formation of a pseudo aneurysm.

CT Angiography has been the gold standard for diagnosing carotid dissection when there is involvement of the cervico-cephalic region. Imaging may reveal dissection of a pseudoaneuysm or an intimal flap from elevation of the intima from extravasated blood, as described in this case. Irregular or smooth tapered narrowing of the lumen may be seen, making it difficult to diagnose on initial imaging [1]. In cases where vertebral artery involvement and subarachnoid hemorrhage is suspected, CT Angiogram should not be replaced with MRA as it is more sensitive to this injury. Sonography is less reliable than CT angiogram and MRA, however it is patient friendly and less expensive. Findings on sonography may consist of echogenic intimal glap and echogenic thrombus, with dissected region located distal to the visualized region on sonogram. Abnormal sonographic findings may also include decreased velocity of the carotid bulb and the presence of high resistance of a biphasic pattern. Carotid dissections typically resolve in more than eighty percent of cases. Recurrence is typically uncommon.

Management is usually nonsurgical, with anti-platelets recommended for presentations of headaches, occulosympathetic paresis (Horner's syndrome), and bruits [2]. In patients with recurrent or progressive transient ischemic symptoms and strokes despite being on aspirin, anticoagulation should be considered.

\section{Case Report}

A 38 year old with a history of tobacco abuse and oral contraceptive use presents to the emergency department with transient loss of vision in the right eye, which lasted for thirty minutes, with a second episode that respected her horizontal meridian. Pt initially developed a nonproductive cough for two weeks, followed by right-sided neck pain prior to the episode of vision loss. Vital signs notable for a blood pressure of $124 / 85$, heart rate $63 \mathrm{bpm}$, and respiratory rate 16 . Eye exam revealed $20 / 20$ vision, $3 \mathrm{~mm}$ pupils, equal and brisk to direct and consensual light, and reactive to accommodation. Cardiopulmonary examination revealed regular rate and rhythm, with a $2 / 6$ systolic murmur loudest at the left lower sternal border, without rubs or gallops and lungs was clear to auscultation. Neurologic examination revealed intact cranial nerves, preserved strength in all extremities. Electrocardiogram revealed normal sinus rhythm, no acute ST changes. Computed tomography of the head was negative. Pt was discharged home with a follow-up appointment with an Ophthalmologist, who noted a normal eye exam with an unremarkable external and retinal dilated exam. The patient was referred to Cardiology for cardiovascular workup. Carotid ultrasound revealed decreased velocity in the right internal carotid artery with evidence of an intimal flap. CT Angiogram of the neck confirmed diffuse narrowing and string sign of the right intracranial internal carotid artery due to carotid dissection (Figure 1). The right ophthalmic artery was not well visualized. The patient was started on aspirin, and tolerated it well without recurrence of symptoms.

*Corresponding author: Kareem Abed, Atlanticare Regional Medical Center, USA, Tel: +1 609-652-1000; E-mail: abedlkareem@gmail.com

Received June 09, 2015; Accepted July 13, 2015; Published July 15, 2015

Citation: Abed K, Misra A, Vankawala V (2015) Spontaneous Internal Carotid Dissection in a 38 Year Old Female: A Case Report. J Clin Case Rep 5: 555 doi:10.4172/2165-7920.1000555

Copyright: ( 2015 Abed K, et al. This is an open-access article distributed under the terms of the Creative Commons Attribution License, which permits unrestricted use, distribution, and reproduction in any medium, provided the original author and source are credited. 


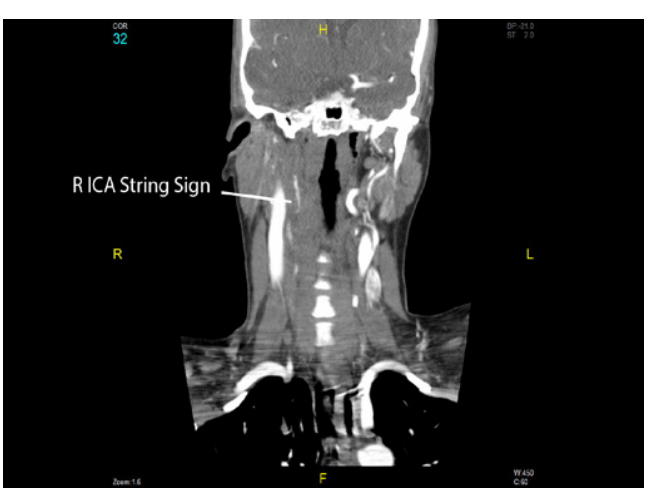

Figure 1: This image shows dissection of the right internal carotid artery, which was the etiology for amaurosis fugax. This is noted as a the string sign, representing the extent of the dissection.

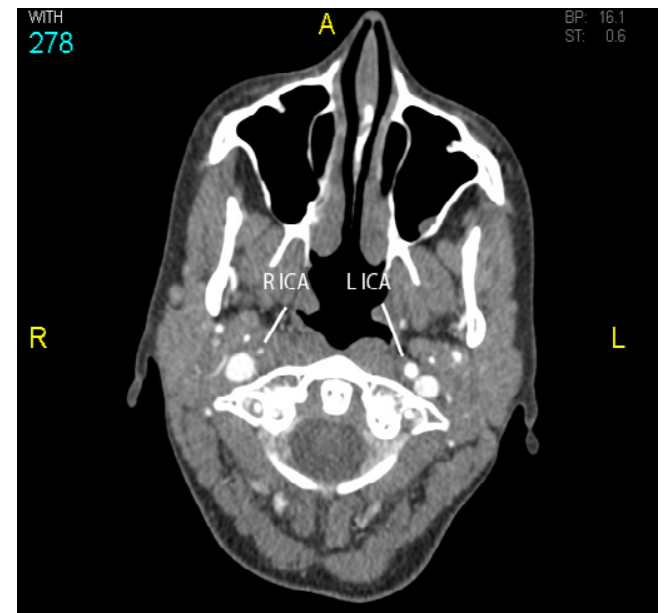

Figure 2: Dissection of the right internal carotid artery is noted in a transverse view.

\section{Discussion}

This case describes the initial signs and symptoms of carotid dissection, with resultant amaurosis fugax. This case stresses the vague symptoms of neck pain and headache (>90\%), followed by focal hemispheric ischemic symptoms (50-90\%), Horner's syndrome $(<50 \%)$, monocular blindness (6-30\%) and dysgeusia (10-19\%) [3]. Given that the most common symptoms are nonspecific, early diagnosis can be difficult, as symptoms are overlooked. In this case, the patient was referred to Ophthalmology and Cardiology before the diagnosis was suspected. Careful attention to the above symptoms will assist in early suspicion and testing for carotid dissection. Early diagnosis and treatment is imperative to prevent the progression of the dissection, which can potentially lead to cerebral infarction, especially in young adults. This case reinforces the importance of a careful history and physical exam, as well as differential diagnosis of monocular blindness in patients presenting to the outpatient office or the emergency department.

Common non-traumatic causes of carotid dissection include fibromuscular dysplasia, Marfan's syndrome, atheroma, cystic medial necrosis, and systemic hypertension. In cases with carotid dissection, hemorrhage into the carotid wall could lead to intimal injury. Hematomas may form as the dissection progresses into the media, which can occlude the lumen. Diagnosis is typically initiated with carotid ultrasound as symptoms raise suspicion for source of possible emboli. Internal carotid artery dissection can produce cerebral infarction in $36-68 \%$ of patient, as occlusion of the lumen near the dissection or embolization of thrombus occurs [3]. An echogenic intimal flap or thrombus, along with decreased velocity of the carotid artery may be noted on Doppler ultrasound. CT Angiogram is the gold stand for diagnosing cervico-cephalic carotid dissection, noting tapered narrowing of the lumen. Patients respond well to anti-platelet agents, with limited recurrence, as this patient did. In cases where recurrence is noted despite appropriate anti-platelet agents, anticoagulation with coumadin may be considered (Figure 2).

\section{References}

1. Provenzale $J(1995)$ Dissection of the internal carotid and vertebral arteries: imaging features. American Journal of Roentgenology 5: 1099-104.

2. Elamel M, Humphrey P, Shaw MJ (1990) Dissection of the cervical internal carotid artery. The role of Doppler/Duplex studies and conservative management. Journal of Neurology, Neurosurgery, and Psychiatry 5: 379-383.

3. Haneline M, Lewkovich (2004) Identification of internal carotid artery dissection in chiropractic practice. The Journal of the Canadian Chiropractic Association 3: 206-210. 